\title{
Simulation of bone indentation
}

\author{
S. Kasiri ${ }^{1}$, G. Reilly ${ }^{1,2} \&$ D. Taylor ${ }^{1}$ \\ ${ }^{1}$ Trinity Centre for Bioengineering, Trinity College Dublin, Ireland \\ ${ }^{2}$ Institute of Technology Sligo, Ireland
}

\begin{abstract}
Finite Element (FE) methods have been widely used to model fractures. The Theory of Critical Distances (TCD) was proposed to predict the fatigue fracture of materials. With the introduction of FE models, this theory has been developed and extensively applied to different materials. FE models of fractures usually need a high resolution meshing or remeshing due to introducing new cracks. This can be a disadvantage, e.g. when the fracture plane of the material is under compression. In this paper the TCD was adopted to predict the multiaxial fracture of bone with the aim of studying the effects of geometry on fracture force.

Keywords: bone, fracture, indentation, multiaxial failure, Theory of Critical Distances.
\end{abstract}

\section{Introduction}

In different surgeries various kinds of blades are used for cutting bone. Bone has a complicated composite structure and many researchers attempt to understand the mechanism of fractures. Understanding the fracture mechanism, cutting process and predicting the fracture load is useful to optimize the surgical blades. To predict the fracture load especially in complicated problems, computer modelling is necessary. Finite Element (FE) methods have been extensively employed to simulate the fracture and cutting process.

The first model of the cutting process using FE obtained the fracture load by simulating the crack's growth until it was an unstable crack. The maximum force before the unstable crack is the cutting force. In a general classification, two different sorts of FE analysis have been proposed to study the crack propagation, the discrete and smeared approaches [1]. The discrete approach deals with the fracture process by introducing a discontinuity in the geometry of the structure 
while the rest behaves in a linear elastic way. The smeared approach keeps the geometry fixed but the strain localization is simulated in a representative region of the structure through the material constitutive law. In the first method, introducing a new crack and remeshing during the analysis is necessary while a high resolution mesh must be made in the second approach. These methods are useful in particular studies of crack growth path. In the case of indentation a developing crack will be closed due to the high compression on the fracture plane. Therefore applying the same methods such as cohesive zone or some discrete models are not appropriate.

In this paper, the Theory of Critical Distances (TCD) is applied to predict effects of indenter geometry on the fracture force. The results were validated using experiments.

\section{Materials and methods}

The Theory of Critical Distances (TCD) was proposed by Whitney and Nuismer [2] to predict the fatigue strength of notched materials. The TCD assumes that fracture occurs when the stress at some characteristic distance, $r_{c}=L / 2$, away from the discontinuity reaches a certain stress. The distance, L, and the certain stress are called the critical distance and critical stress respectively. This theory has been recently developed to be applicable to brittle fracture as well as fatigue [3] for various materials [4, 5].

As most engineering components are subjected to a complex loading system, the TCD has been extended for multiaxial stress fields [6]. For multiaxial TCD an equivalent critical stress should be defined. It is mostly a function of shear and normal stress acting on the fracture plane. The criterion depends on the failure criterion of the material. The failure mechanisms differ for different materials; therefore, some critical stress must be defined for a given material under multiaxial loading state.

Indentation is a multiaxial problem. A complex field of stress including shear and normal stresses is acting on the critical plane. Here, to study the bone fracture, the Columb-Mohr criterion was employed which was proposed for brittle multi-axial fracture. It is assumed that the critical stress is a linear combination of the normal and shear stress on the fracture line as shown in Equation (1). The critical stress is assumed to be the shear strength, $\tau_{\mathrm{u}=72 \mathrm{MPa}}$, as the equation is satisfied if there is no normal stress.

$$
|\tau|+\alpha \cdot \sigma=\tau_{u}
$$

$\alpha$ is a material constant, which depends on the relative strength of material under different stress states. $\alpha$ and L are two unknown material constants and so can be obtained from two sets of experimental data. Thus to apply the TCD, first two sets of data were employed to find the material constants and then these have been used to predict the indentation force for other indenter geometries. 
Indentation tests were carried out on $8 \mathrm{~mm}$ cubes of cortical bone from bovine femora, indented in two directions with different blades, either parallel or perpendicular to the bone's longitudinal axis. The indentation was performed at a rate of $2 \mathrm{~mm} / \mathrm{min}$ at room temperature. Indenters with different angles and radii were used and they were lubricated so as to reduce the friction between materials.

A 2D finite element model of was established using ANSYS software. Bone was modelled as a nonlinear isotropic material, the indenter as a rigid material. Load was applied to the indenter as a force, deformation was determined to validate the model. All nodes on the bottom surface of the bone sample (and along the sides for the lower $1 \mathrm{~mm}$ ) were constrained in the vertical direction. A map mesh using element PLANE82 was used around the contact area. High deformation around the contact area tended to cause a mesh destruction error. To avoid this problem a mesh ratio up to $1: 2$ was used in this region. The contact surfaces were modelled using CONTA172 and TARGE169 with Standard behaviour. The penalty method was employed as a solver.

\section{Results}

Experiments showed that the crack initiates and grows in the longitudinal direction of the bone, which is roughly the same as the osteons' orientation, and the weakest direction in the bone. It is independent of the indenting direction. Figure 1 shows the main crack growth in both directions of indentation, longitudinal and transverse. A critical plane is therefore defined in the longitudinal direction starts from crack initiation spot.
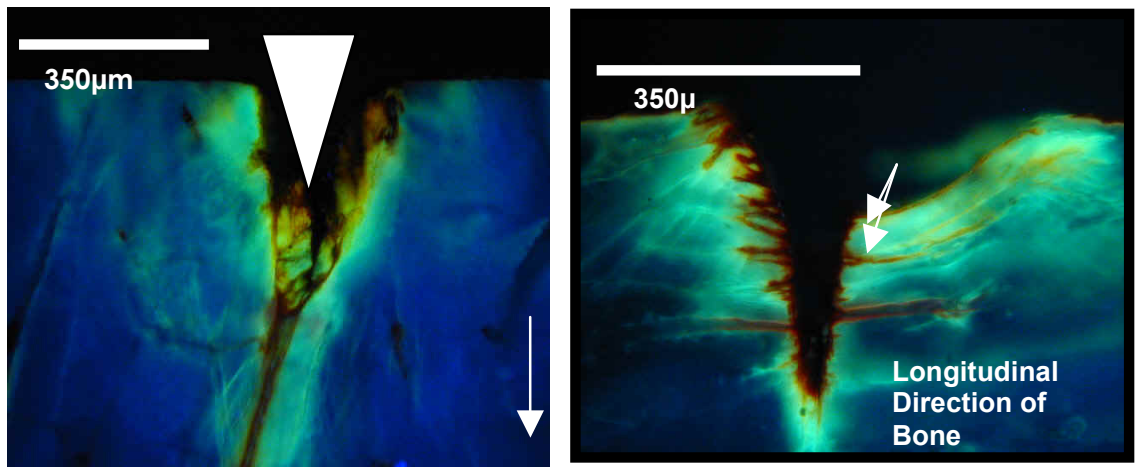

Figure 1: Crack direction during longitudinal (on the left) and transverse (on the right) indentation. In both cases cracks develop in the longitudinal direction.

The crack initiates from the most highly loaded spot on the blade/bone interface. Assuming bone is a homogenous material under indentation makes it easier to define the crack initiation place. There are two different types of 
singularities in a blade, boundary and geometric singularities. The first is defined as the place where the indenter is separated from the material. This occurs due to the sudden change in pressure on the material from a finite value to zero. This singularity depends on the contact area between two bodies and also their material properties. The second singularity occurs at the point along indenter geometry where the radius of curvature changes. In fact even for a perfect indenter, with smooth sides, the radius of boundary curvature is changed from a finite value at the tip to an infinite value along the sides. This variation in the curvature causes a singularity during the contact due to changing the pressure curve on the contact surface. Figure 2 shows the singularities on the contact surface. As can be seen the normal stress on the contact surface changes markedly as a result of shifting on contact pressure at singular points. Singularities in stress during the indentation are probable locations for crack initiation. In the case of longitudinal indentation the geometric singularity plays an important role. In a special case, if the radius of the indenter tip goes to zero then the crack starts from the tip, which is the geometric singularity.

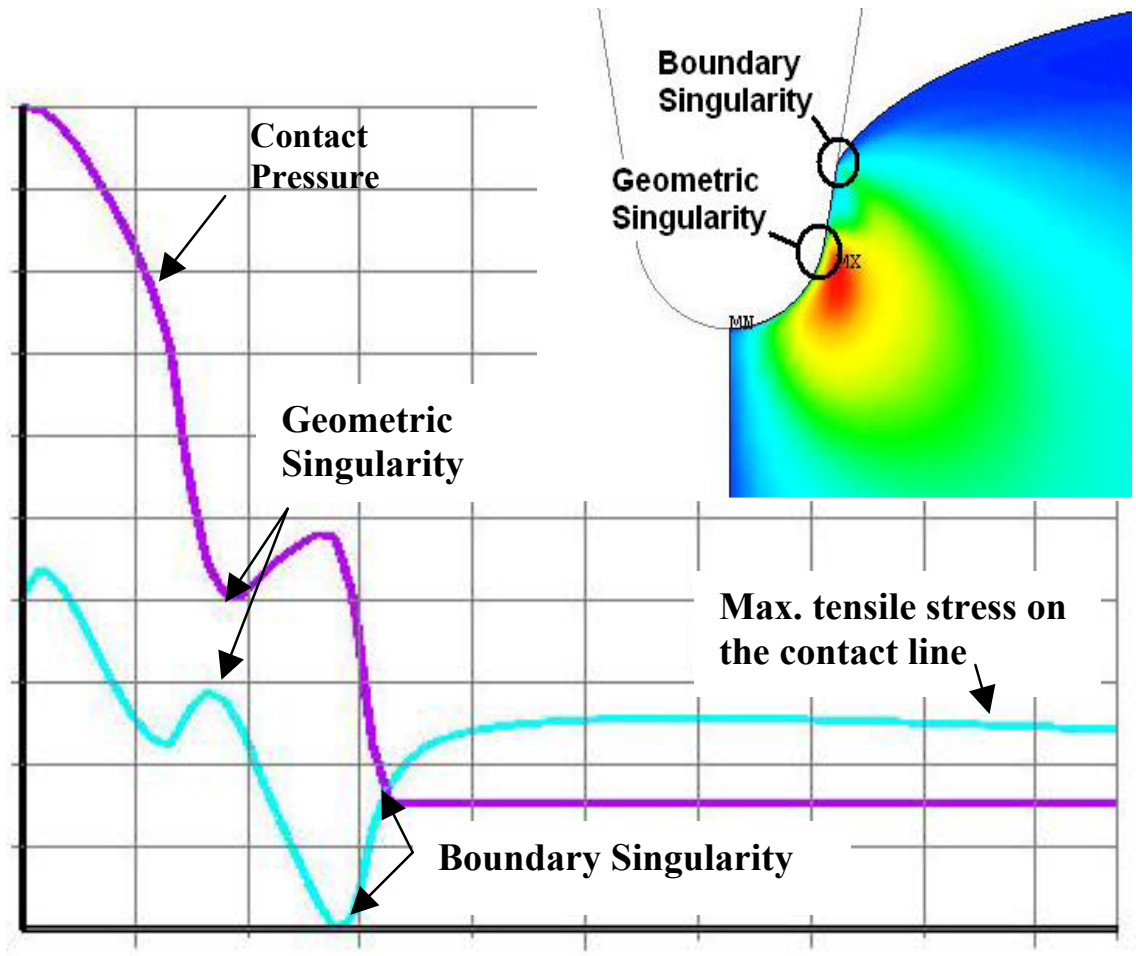

\section{Distance}

Figure 2: $\quad$ Schematic of the variation of first principal stress (S1) and contact pressure during indentation and effects of the singularities. 
Figure 3 shows the variation of shear and normal stress on the fracture line for longitudinal indentation. It demonstrates that on the fracture line the material is highly under compression. According to eqn. (1) a high shear stress is required for failure.

Using the experimental results for two different indenters $\left(20^{\circ}-300 \mu \mathrm{m}\right.$ and $\left.20^{\circ}-700 \mu \mathrm{m}\right)$, and the defined critical line the critical distance for bone was estimated to be $0.32 \mathrm{~mm}$. The value of $\alpha$ also calculated to be 0.3 . These values of critical distance and $\alpha$, assumed to be material constants, were employed to predict the fracture loads in the other tests. The fracture line, on which calculations were performed, was assumed always to be longitudinal.

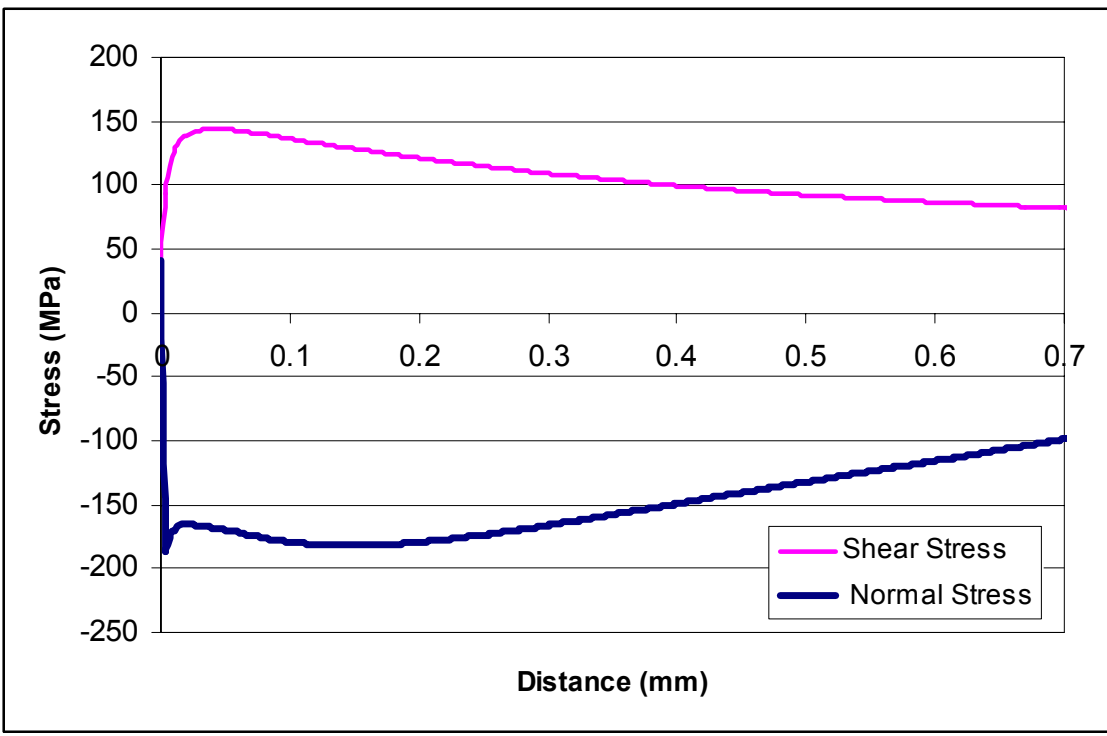

Figure 3: Variation of normal stress and shear stress on the critical line for $20^{\circ}-700 \mu \mathrm{m}$ indenter at the fracture force.

Figure 4 shows data and predictions of the effect of tip radius on fracture force for a constant indenter angle of $20 \mathrm{o}$, for both longitudinal and transverse indentation. The results show that there is an increase in fracture force with the radius of indenter, the rate of increase being greater at lower radii. Also the fracture force in the transverse direction is larger than that in the longitudinal direction. The TCD can predict these results well.

The experimental results showed only a small variation on fracture load with angle for large radii. For example, for indenters with radius of $700 \mu \mathrm{m}$, the failure force changed from $2678 \pm 156(\mathrm{~N})$ to $2834 \pm 380(\mathrm{~N})$ when the angle changed from $20^{\circ}$ to $60^{\circ}$. The TCD predicts the same force for both indenters, with a value of $2700 \mathrm{~N}$. For smaller radii the TCD predicts significant variation in force with angle; predicted force changes from $921(\mathrm{~N})$ to $1180(\mathrm{~N})$ for 50 Micron blades when the angle changes from $20^{\circ}$ to $60^{\circ}$. 


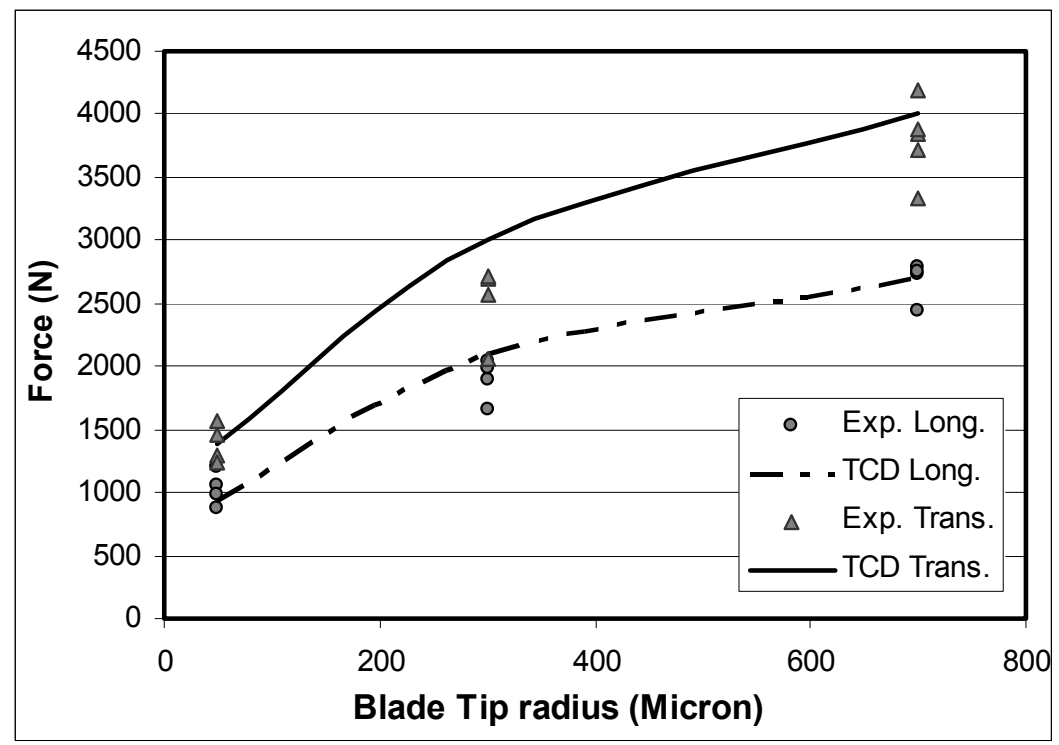

Figure 4: Experimental results and TCD predictions of cutting force for blades with $20^{\circ}$ angle and various radii.

\section{Discussion}

Under indentation, failure occurs as a result of normal and shear stress on the fracture plane. In this case the normal stress is mostly compression especially for blunt indenters. It is shown in Figure 3 that in the presence of compression the shear stress is much higher than the shear stress necessary to cause fracture. Eqn. (1) is the simplest application of the Coulomb-Mohr criterion. According to this theory fracture occurs on a given plane in the material when the critical linear combination of normal and shear stress acts on this plane. According to this criterion $\alpha$ and $\tau_{u}$ are material constants that are related to ultimate stresses in tensile, compression and shear. Assuming the typical values of bone for compression, tensile and shear ultimate stress to be about $-250,150,68 \mathrm{MPa}$ respectively gives $\alpha=0.35$ and $\tau_{\mathrm{u}}=72 \mathrm{MPa}$. Using the critical distance method and the experimental results for two groups of data, $\alpha=0.3$ was estimated which is therefore comparable with the Coulomb-Mohr constant.

Fracture properties of bone vary in a wide range according to the literature [7-20]. The variation in fracture properties stems from the different testing conditions and different types of bone is used. The fracture characteristics of bone are a function of its orientation and are notch sensitive [21]. The Critical Crack Length is a material property, which is defined as the size of the largest crack that a material can tolerate before unstable fracture occurs. This value of critical crack length is similar to our critical distance value. Some research has been performed to obtain the critical crack length of bone. In the literature the 
critical crack size of bone has been found to vary between $0.3 \mathrm{~mm}$ and $1.8 \mathrm{~mm}$ $[10,12,15,16]$. Bonfield and Datta [10] looked at tensile specimens of bovine femora containing transverse cracks on the edge and centre. They found a Critical Crack Length of $\mathrm{L}=0.37 \mathrm{~mm}$. Robertson et al [16] studied V-notched specimens of bovine bone using three-point bending. The critical crack length perpendicular to the tensile stress axis was determined to be $0.36 \mathrm{~mm}$ at the strain rate of $7 \times 10^{-6}$ to $3 \times 10^{-2} \mathrm{sec}^{-1}$. Moyle and Gavens [12] analysed bovine plexiform tibia with different crack lengths $(4-12 \mathrm{~mm})$ and tip radii in transverse direction. They found the critical crack length to be $1.8 \mathrm{~mm}$ but Kc was overestimated about $11.2 \mathrm{MNm}^{-1 / 2}$. It is also interesting that the fracture toughness of human bone and bovine bone relative to its strength are approximately similar [14]. In the other words, the critical distances of bovine and human bone are roughly similar. We estimated $\mathrm{L}$ be about $\mathrm{L}=0.32 \mathrm{~mm}$ for longitudinal bovine femora. This value of critical distance is comparable with the critical crack length reported in other literatures.

For the blunt indenters, the radius has a large effect on the fracture force in both indenting directions, longitudinal and transverse. The larger the radius the larger the fracture force. This is due to the increase of the contact area when increasing the radius. Increasing the radius from $50 \mu \mathrm{m}$ to $700 \mu \mathrm{m}$, changes the longitudinal fracture force from about 1000 to around 3000 . It is quite interesting, that there is not a major difference between the fracture load of a sharp blade and a blade with $50 \mu \mathrm{m}$ radius.

For indenters with small radii, the fracture force increases when increasing the angle. The experiments showed that the larger the radius the less effect of angle on the fracture force. Our analysis showed that, for a large enough radius of indenter, the edges of indenter that carry the effects of the angle are not in contact with the bone before fracture. Therefore only the radius has an effect on the fracture force. But for small radii, there is enough deformation before the fracture such that the sides of the indenter play a role.

\section{Conclusions}

1. The Theory of Critical Distances was able to predict the effects of radius, angle and loading direction on the indentation fracture force in bone.

2. It demonstrated that the radius has more effect than the angle, and that both effects diminish with increasing radius.

3. The effect of material anisotropy was successfully accounted for simply by changing the direction of the critical line (predicted fracture line).

4. The approach is very easy to implement using FEA: modelling of crack growth is not necessary, which is a great advantage when predicting failure under compressive loading.

\section{Acknowledgement}

This work was funded by the Irish Council of Directors Strand III Research Programme 


\section{References}

[1] de Borst, R., Remmers, J.J.C., Needleman, A. \& Abellan, M.A., Discrete vs smeared crack models for concrete fracture: bridging the gap, International Journal for Numerical and Analytical Methods in Geomechanics, 28(7-8), pp. 583-607, 2004

[2] Whitney, J.M. \& Nuismer, R.J., stress fracture criteria for laminated composites containing stress-concentrations Journal of Composite Materials, 8, pp. 253-265, 1974

[3] Taylor, D., A mechanistic approach to critical-distance methods in notch fatigue, Fatigue \& Fracture of Engineering Materials and Structures, 24(4), pp. 215-224, 2001

[4] Taylor, D., Predicting the fracture strength of ceramic materials using the theory of critical distances, Engineering Fracture Mechanics, 71(16-17), pp. 2407-2416, 2004

[5] Taylor, D., Merlo, M., Pegley, R. \& Cavatorta, M.P., The effect of stress concentrations on the fracture strength of polymethylmethacrylate, Materials Science and Engineering A, 382(1-2), pp. 288-294, 2004

[6] Susmel, L. \& Taylor, D., Two methods for predicting the multiaxial fatigue limits of sharp notches, Fatigue \& Fracture of Engineering Materials and Structures, 26(9), pp. 821-833, 2003

[7] Behiri, J.C. \& Bonfield, W., Crack velocity dependence of longitudinal fracture in bone, Journal of Materials Science, V15(7), pp. 1841-1849, 1980

[8] Behiri, J.C. \& Bonfield, W., Orientation dependence of the fracture mechanics of cortical bone, Journal of Biomechanics, 22(8-9), pp. 863867,1989

[9] Bonfield, W., Advances in the fracture mechanics of cortical bone, Journal of Biomechanics, 20(11-12), pp. 1071-1081, 1987

[10] Bonfield, W. \& Datta, P.K., Fracture toughness of compact bone, Journal of Biomechanics, 9(3), pp. 131-132, 1976

[11] Lucksanasombool, P., Higgs, W.A.J., Higgs, R.J.E.D. \& Swain, M.V., Fracture toughness of bovine bone: influence of orientation and storage media, Biomaterials, 22(23), pp. 3127-3132, 2001

[12] Moyle, D.D. \& Gavens, A.J., Fracture properties of bovine tibial bone, Journal of Biomechanics, 19(11), pp. 919-927, 1986

[13] Nalla, R.K., Kinney, J.H. \& Ritchiei, R.O., Mechanistic fracture criteria for the failure of human cortical bone, Nature Materials 2, pp. 164-168, 2003

[14] Norman, T.L., Vashishth, D. \& Burr, D.B., Fracture toughness of human bone under tension, Journal of Biomechanics, 28(3), pp. 309-320, 1995

[15] Piekarski, K., Fracture of Bone, Journal of Applied Physics, 41(1), pp. 215-223, 1970

[16] Robertson, D.M., Robertson, D. \& Barrett, C.R., Fracture toughness, critical crack length and plastic zone size in bone, Journal of Biomechanics, 11, pp. 359-364, 1978 
[17] Vashishth, D., Behiri, J.C. \& Bonfield, W., Crack growth resistance in cortical bone: Concept of microcrack toughening, Journal of Biomechanics, 30(8), pp. 763-769, 1997

[18] Wright, T.M. \& Hayes, W.C., Fracture mechanics parameters for compact bone--Effects of density and specimen thickness, Journal of Biomechanics, 10(7), pp. 419-425, 1977

[19] Yan, J., Clifton, K.B., Mecholsky, J.J.J. \& Gower, L.A., Effect of temperature on the fracture toughness of compact bone, Journal of Biomechanics, In Press, Corrected Proof (pp. 2006

[20] Yan, J., Clifton, K.B., Mecholsky, J.J. \& Reep, R.L., Fracture toughness of manatee rib and bovine femur using a chevron-notched beam test, Journal of Biomechanics, 39(6), pp. 1066-74, 2006

[21] Bonfield, W. \& Li, C.H., Deformation and Fracture of Bone, Journal of Applied Physics, 37(2), pp. 869-875, 1966 